\title{
The Older Women with Urinary Incontinence and Chronic Illness Who Living in Urban Community: A Case Study
}

\author{
Supichaya Wangpitipanit ${ }^{1 *}$, Supunnee Thrakul $^{2}$ and Satakamol Prasongwattana ${ }^{3}$ \\ ${ }^{1}$ Lecturer, Ramathibodi School of Nursing, Faculty of Medicine Ramathibodi Hospital, Mahidol University, Bangkok, Thailand \\ ${ }^{2}$ Assistant Professor, Ramathibodi School of Nursing, Faculty of Medicine Ramathibodi Hospital, Mahidol University, Bangkok, Thailand \\ ${ }^{3}$ Registered Nurse, Bang Pa-In Hospital, Phra Nakhon Si Ayutthaya Province, Thailand \\ *Corresponding Author: Supichaya Wangpitipanit, Lecturer, Ramathibodi School of Nursing, Faculty of Medicine Ramathibodi Hospital, \\ Mahidol University, Bangkok, Thailand.
}

Received: June 11, 2019; Published: July 11, 2019

DOI: $10.31080 /$ ASNH.2019.03.0363

\begin{abstract}
Urinary incontinence is caused by loss of bladder control and involuntary leakage of urine. It can have a health problem such as physical, psychological, social interaction and significant impact on the quality of life. This case was designed to study the nursing care outcomes for older women with urinary incontinence and chronic illness (hypertension, dyslipidemia, spinal stenosis L3-4, and chronic kidney disease stage 4) who living in a community located in Bangkok by approach Donabedian conceptual framework including structure, process, and outcomes of quality of care. There were 4 outcomes as managing urinary incontinence, self-care pressure ulcers, rehabilitation, and helping with early depression symptoms. Four times for home visits within 3 months for nursing care were composed of Foley's catheter care with bladder training, pelvic muscle exercise, dressing wound from incontinenceassociated dermatitis, arm-shoulder exercises following the manual of self-care, and finally adjust the home environment to install the fixed pulley. Results presented the older women could be to improve symptom: no leakage of urine, dermatitis and pressure ulcer decreased, improve shoulder mobility and walking. Suggestions should be provided systems for home visiting to assess and monitors for self-care continuously.
\end{abstract}

Keywords: Older Women; Urinary Incontinence; Chronic Illness; Urban Community; Case Study

\section{Introduction}

Urinary incontinence (UI) is a common problem of an older woman which cannot control urinary excretion by loss of bladder control and involuntary leakage of urine lead to a health problem such as physical, psychological, social interaction and significant impact on the quality of life [1-3]. It almost occurs as incontinenceassociated dermatitis (IAD) that is the skin folds at groin area by exposing urine for a long time causes to redness dermatitis [4]. In addition, there is a chance of decubitus ulcer, pressure sore, urinary tract infection, and fall from an accident causing loss of ability to perform daily activities [5]. Urinary incontinence also causes psychological problems and social involvement that shameful from the smell often make traveling more difficult. The older woman with have many problems from urinary incontinence are often separated from society causing depression and it also affects the economic conditions in the family by paid the device to support the urine leakage, therefore inevitably will cause health effects in all aspects that lead to reduced quality of life [1-3,6].

The prevalence of the study found that the older woman with urinary incontinence in the community was as high as 12-15\% [7]. The women were twice as much as the older men [2,3]. Causes of urinary incontinence were increasing from the old age of degenerative, acute event from an accident at spinal cord injuries, chronic disease or illness. Chronic illness refers to the suffering or condition of the person in physical and mental discomfort and the cause and effect of the disease that has already been caused by an illness 
that cannot be healed [8]. The older woman with urinary will have comorbidities from chronic non-communicable diseases such as cardiovascular disease, diabetes, high blood pressure, paralysis, heart disease, asthma, chronic bronchitis, and etc. [1-4].

The first approach is to manage these symptoms such as using urine pads, adult diapers, behavior modification, and behavioral therapy. Behavior modification is changing the active lifestyle in daily living such as drinking of water; avoid drinking caffeine, weight control body mass index (BMI) to normal, prevention of constipation. In the part of behavioral therapy is used for improving bladder control such as bladder training by practice to prolonging urinary excretion in a period time, and pelvic-floor muscles exercise (Kegel exercise) involves repeatedly contract and relax in order to strengthening pelvic-floor muscles can be control of urination. It is necessary for cannot control urination, should have a diagnosis for treatment such as receiving drugs, wearing a vaginal pessary ring, receiving urinary and vaginal surgery until intermittent catheterization or indwelling urinary catheterization [1-3,6]. The older women with urinary incontinence faced with many problems mentioned above inescapably, therefore, should promote self-care ability that covers physical, mental, social, economic dimensions from interdisciplinary healthcare provider team. Especially the profession nurses as health service providers are an important person in caring for the individual, family, and the community. Nurses will continue to find an older woman with urinary incontinence at home or in the communities that have living and affecting to worse quality of life by providing nursing care according to problems and needs, promoting knowledge in selfcare, increasing and practicing the skills necessary for rehabilitation to prevent resulting in more complications. Therefore, the reason this study is to evaluate the results after the nursing care outcomes for older women with urinary incontinence and chronic illness living in a community.

\section{Case study}

Home visited an older woman with urinary incontinence and chronic illness, aged 72 years, widow status, Islam religion, graduated in the fourth year of study that lived in the urban area at Bangkok. The detail of chief complaint, she had a rash on the genital area for 2 weeks ago. In the past of history, nineteen years ago, she had been falling accident gathering surgery spinal cord injuries at L3-4 causing the problems of movement and balance effecting to decrease activities daily living. Later from two years ago, she got a being detected as high blood pressure, dyslipidemia, and chronic kidney disease by treatment continuous medication. One year ago, she began to have symptoms of urinary leakage type of overflow urinary incontinence unable to control urinary excretion, after that she received many treatments at the hospital based on the urinary catheter. In the past of family history, her husband died of heart disease twenty years ago. There are four children (two men and two women), and the oldest daughter which is the primary caregiver died from diabetes for two months ago. She gave information that felt like alone and lack of care when her sick. Now, she had a young daughter who is not bound together because of her younger brother had been raised since she was young by her younger daughter will buy supplies for food every week and take her to the hospital by receiving money from a younger son amount 1,000 baht per month. The older woman who is urinary incontinence and chronic illness lived in a crowded city. The house way is a small alley by walking together approximately 300 meters in length, with garbage along the side. The environment inside the house is two floors single-family wooden, the upper floor is wood with stairs outside, the ground floor is a cement and a small room with an ensuite bathroom which have a condition is quite moist and has a musty smell and the air is not flowing that she could do the instrumental of daily activities as cooking or doing religious activities in the room at home. Every evening she would have had activities of the community by slowly walking to the wall from the alley to join and exercise.

Health assessment and results

GA: Thai female old age, good consciousness, neuropathic gait demonstration

VS: $\mathrm{T}=37^{\circ} \mathrm{C}, \mathrm{PR}=88 / \mathrm{min}, \mathrm{RR}=20 / \mathrm{min}, \mathrm{BP}=160 / 90 \mathrm{mmHg}$, repeated $\mathrm{BP}=140 / 90 \mathrm{mmHg}$

Body weight: 62 Kgs., Height $151 \mathrm{~cm} . \mathrm{BMI}=27.2 \mathrm{Kg} / \mathrm{m} 2$

HEET: Mouth and Throat: No dental caries, mild injected pharynx, tonsils not enlarged, no exudate

Chest: Symmetry, normal contour, AP: transverse approximately 5:7, chest wall no lesion, equal movement

Heart: PMI at 5th Lt, ICS and MCL, normal S1S2, no murmur HR: 90/min, strong and regular 
Lungs: Equal expansion, normal \& equal breath sound, no adventitious sound

Abdomen: Bound sound 10 times/min, soft, no tenderness, no palpable liver or spleen

Back: No scoliosis or lordosis, no tenderness both CVA

Neurological Signs: Level of consciousness alert, well cooperated and good orientation to time, place and person, normal muscle tone, motor power grade $\mathrm{V}$ all extremities exception at both legs grade IV

\section{Reflexes: DTR 2+ all}

Genitalia: Rash at perineum, moderates IAD

Others: Decubitus ulcer at buttock and coccyx stage I

Thai Geriatric Depression Scale (TGDS-15) = Score 6

Barthel activity daily living index score $=11$

\section{Problem list}

- Abnormal gait and posture: neuropathic gait demonstration

- Hypertension BP = 160/90 mmHg and 140/90 mmHg after repeat in $15 \mathrm{mins}$.

- Mild injected pharynx

- $\quad$ Motor power grade IV at both legs

- Rash at perineum: moderates IAD

- Decubitus ulcer at buttock stage I

- Retained catheterization with urine bag: turbid urine

- $\quad \mathrm{BMI}=27.2 \mathrm{Kg} / \mathrm{m} 2$ (Obesity level 1 ) [9]

- Barthel ADL index score 11 (intermediate initial score, moderately severs dependence) [10]

- $\quad$ Thai Geriatric Depression Scale (TGDS-15) [11,12] Score 6 (began to have depression)

Chronic diseases: hypertension, spinal stenosis L3-4, and chronic kidney disease stage 4

- Lab chemistry: Blood urea nitrogen $=35(7.0-18.0 \mathrm{mg} / \mathrm{dl})$

- Creatinine 1.95 (0.51-0.95 mg/dl)

- $\quad$ eGFR (CKD-EPI $24.2 \mathrm{ml} / \mathrm{min} / 1.73 \mathrm{~m}^{2}$ )

Treatment plan: Dressing wound IAD and decubitus ulcer OD.

- $\quad$ Retained Foley's catheter No. 16 with urine bag

- Amlodipine (10 mg) 1tab oral OD p.c.

- $\quad$ Folic acid 1 tab oral OD p.c.
- $\quad$ FBC 1 tab oral tid p.c.

- $\quad$ Sodamint 2 tab oral bid p.c.

- $\quad$ Simvastatin (20 mg) 1 tab oral OD h.s.

\section{Discussion}

From the details of health assessment and results of the physical examination found that an older woman with urinary incontinence and chronic illness have many significant problems both physical and mental health including lifestyle daily living effecting to worse quality of life, therefore the necessary interdisciplinary care team approach particularly the professional nurse must be managing caring by summarizing incontinence-associated dermatitis (IAD), urinary incontinence (UI), activity daily living (ADL) problems, chronic illness management, and depression are follows as:

- Incontinence-Associated Dermatitis (IAD): The older women with urinary incontinence will have leakage of urination causing skin inflammation due to incontinence associated dermatitis (IAD). IAD is a condition of loss of moisture in the skin from prolonged contact with urine or feces $[4,11$ 13]. The main cause of the destruction of the epidermal layer to the dermis seen as a rash on the perineal and groin area, which is likely to be infected by bacteria and fungi can classify the severity of dermatitis into 5 levels as follows: (1) High risk to IAD: The stage to the risk of dermatitis to the skin is not red with normal temperature, not warm, not swollen or hot frequently found in a bedridden older person, unable to communicate or control excretion usually most common seen often associated with diarrhea, (2) Early IAD: The stage of beginning to dermatitis, normal-dry skin, no blister, clear water or blister, the skin area is pink or red by not seem scope is not clear. The older person will complain when the wound is touched, (3) Moderate IAD: The stage of moderate skin inflammation lacks moisture, with bright red color, bleeding spots or blistering. The older person will complain of pain when touching the wound area, (4) Severe IAD: The stage of severe dermatitis skin has a shallow, red wound, with blood seeping into the open wound on the upper skin, peeling off. An older person will always complain of her pain and (5) Fungal-appearing rash IAD: The stage of skin inflammation and fungal infections has a fungal rash, white or yellow, at the edge of the wound likely acne or red spots. The older person will feel itchy and complaining of pain. 
- This case study had the skin inflammation with moderate to the severity level of incontinence-associated dermatitis which found that most common with an older person with urinary incontinence by exposing urine at perineal and groin areas all times. Normally, the skin of a person has weak acidity while urine and feces are alkaline, when the acidic skin is exposed to urine at all times or just 5-15 minutes, also being friction with clothes or mop pads will cause irritation to the skin, loss of moisture the skin is easily peeled, scratched and wound resulting in the growth of microorganisms is the cause of odors [12].

- Urinary incontinence (UI): The older women with problems with urinary incontinence cannot control the excretion at any time. The history and physical examination in this case report showed as overflow incontinence and functional incontinence can urinate before entering the toilet $[1-3,5]$. The urinary excretion mechanism is caused by the interaction of the central nervous system from the brain and spinal cord to the sympathetic peripheral nervous system (hypogastric nerve, T11-L2) and the parasympathetic (pelvic nerve, S2-4) by urine to control the external urethral sphincter to be ready to urinate. When the bladder receives about $150-200 \mathrm{ml}$ of full urine, more pressure in the bladder will occur. The lower end of the bladder nerve is stimulated and afferent impulse through the spinal cord, sent directly to the central urinary excretion control micturition to interpret the effect of urinary pain for voiding. If her in a place not ready to urinate, the brain will send signals from the basal ganglia and the frontal lobe to stop the bladder muscles from contracting allowing further urination. If he went to the bathroom or in the right place for urinary excretion, the central brain will instruct the efferent impulse to come to the bladder to contract together with the outer sphincter of the urethra to release residual urine. In this case of an older woman who had the urethral sphincter dropped more than usual. The mechanism of the sphincter is not strong enough resulting in urinary incontinence pouring out. In addition, the urination does not come out, there is residual urine. There were many factors causing of urinary incontinence including chronic diseases, having a history of having a spinal bone surgery to spinal stenosis to restrict on body movement, overweight, the transmission of nerve impulses in the bladder muscles to be incomplete resulting in the eventual incontinence of urine. Finally, this case report could not control of voiding gathering retained Foley's catheter no.16 Fr. with urine bag. Later, she met the urination leakage again that she went to nearby the hospital receiving treatment again and to change the urinary catheter the more size of no. 20 Fr. with urine bag.

- Moderately severe dependence and chronic illness: The history of this a case report used to have an accident and surgery at the spinal cord to lumbar 3-4 causing problems in movement and balance within an assessment of the Barthel ADL index score is intermediate initial score as at moderate level. The results of the physical examination found that both legs were a motor weakness, abnormal of the nervous system and muscles the body cannot force the muscles to move or function normally affecting movement cannot sit, cannot stand or walk normally with the central nervous system and peripheral nervous system. The central nervous system (CNS) consists of the brain and spinal cord and peripheral nervous system (PNS) consists of the anterior horn cell, nerve root, and nerve, muscle, and joints between nerves and neuromuscular junction (NMJ). Pathological conditions in any part of the nervous system both in the central nervous system and peripheral nervous system will result in weakness organ bring out the problem of body movement affecting the need to rely on daily activities. In addition, there are many factors that affect the movement of the body at various areas including the history of surgery in the spinal cord, together with overweight standard criteria body mass index as an obesity level 1 (BMI $=27.2 \mathrm{Kg} / \mathrm{m}^{2}$ ), resulting in the balance of irregular walking and the strength of the leg muscles and she still has a disease hypertension and high blood cholesterol which has forwarded pathological changes in the vessels, brain, and kidneys found that to be chronic kidney disease stage 4 . Then, estimated from higher laboratory chemistry CKD-EPI value is $24.2 \mathrm{ml} /$ min $/ 1.73 \mathrm{~m}^{2}$ which between 15 to 29 [13] entering kidney failure stage 4 is a condition with continuous renal function deterioration and preparing to enter the renal therapy and replacement system.

- Beginning to have depression: This case report was assessed of the depression test in Thai elderly (TGDS-15) resulting in began to have depression. Depression is an emotional change that affects the mind and body especially an older person will have emotional changes, will feel tired, feel sad, the discouraged inability of activity daily living $[11,12]$. By the way, this 
case report was evaluated to score 6 from Thai language version developed by Nahathai Wongpakarn [11] is a simple and appropriate evaluation form for an older person to self-assess. There were consists of 15 questions as questions about satisfaction with lifeless activity or less attention to things, feel that your life is empty, feeling bored frequently, for example, giving 1 score, questions, answers, yes and no interpretation of results as follows: score 0-4 no depression, score 5-10 beginning to have depression should receive basic advice, and score 11-15 a depressive disorder should see a doctor for treatment, This case report, faced with the loss of the oldest daughter who lived together for a long time, when conversation and listening to the many problems, she said that "feeling like being alone" as well as evaluating depression from TGDS-15 evaluated score 6 , should be continuously monitored and monitored for depression.

\section{Expected outcomes}

The professional nurse visited home the family of the older women with urinary incontinence and chronic illness to 4 times for 12 weeks period, introduced to clarify home visit objectives to assess health and provide nursing care for the urinary catheter together with urinary excretion by following her problems by approach the Donabedian conceptual framework to the use for care management to promote self-care, quality of care of caregivers, and leading to improve quality of life both case report and family caregivers which consists of 3 components (structure, process, and outcomes) following as:

- $\quad$ Structure: It was using tools of physical characteristics and nursing activities including a manual wound and urinary catheterization care, behavioral therapy video media management, the equipment teaching media and practice skills wound dressing at home, the set wound with $0.1 \%$ TA cream, and adjusting the home environment to install the fixed pulley.

- Process: It was a method to conduct nursing activities by giving knowledge, skills, and nursing care through the nursing process including health assessment, diagnosis problems, planning for nursing therapeutic, and evaluation outcomes. This case report had many problems in performing daily activities at a moderate level of dependence, urinary incontinence causing pressure ulcers and troublesome body move- ments. In the role-based process, nurses must assist teaching caregivers to help replace daily routines, urinary catheter care, caring for IAD due to inability to control excretion and enhancing equipment the fixed pulley to facilitate the routine of the older woman to be able to do all the work resulting in good nursing outcomes, reducing complications that have the opportunity to receive hospital treatment $[18,19]$.

- Outcome: It was an affects the quality of good care by determining the results of this nursing care. The activities had determined the results of 4 nursing matters including the management of urinary incontinence, decubitus ulcer care, and self-management in body exercise and walking. After taking care services from the medical team, giving diagnosis and treatment, dealing with urinary incontinence, providing chronic medicine and the physical therapist teams helped training in body exercise and walking in the hospital The older woman was evaluated from the professional nurse and found no leakage of urine, dermatitis and pressure ulcer decreased, improve shoulder mobility and walking were follows as: (1) the problems of urinary incontinence, the profession nurse taught how to clean the genital and take care when properly inserting a urinary catheter, teaching pelvic floor muscles exercise, teaching media and video media practice contract and relax the pelvic floor muscles 5-10 seconds to 25 cycles for 3 times 6 weeks, bladder training and prompt voiding to train practice urinary excretion for a period of time urinate the catheter every 4 hours for 6 weeks. Finally, this case report could not control of voiding gathering retained Foley's catheter with urine bag no.16 French (Fr.). Later, she met the urination leakage again that she went to nearby the hospital receiving treatment again and to change the urinary catheter with urine bag the more size of no. 20 Fr. and found that no urinary incontinence leakage again, (2) teaching skills wet dressing wounds and infection prevention by supporting the wound dress at home from set dressing wound and using external medicine $0.1 \%$ TA cream. At the end of the fourth visits, no red rashes were found in the groin and reproductive organs with finding that the size of the wound decreased, (3) in activities of movement and exercise by learning to walk at home, the profession nurse had helped install the fixed pulley. Exercise the arms and body movements in the middle of the house to practice daily administration 3 times a day. It was found that from the past, 
patients had to leave the house difficult Until able to stick to the edge of the walkway to do community activities outside the house, and (4) helping with early depression symptoms was to assess and plan care, opportunity to drain feelings, focus on promoting selfworth, and coordinate family in psychological care. At the end of the fourth visits, there was no depression.

\section{Conclusion}

This case study is an older woman with urinary incontinence problem caused by an accident in the spinal stenosis L3-4 affecting the ability to perform daily activities decreased and incomplete, a rare case phenomenon for the family and the urban community. An older woman with overflow urinary incontinence is many problems from incontinence-associated dermatitis, decubitus ulcer, right frozen shoulder with the problem of chronic diseases that require continuous care, including high blood pressure and chronic kidney disease stage 3. This case was having a history to be hospitalized with frequent urinary tract infections, facing problems starting with depression from loss of primary caregivers. Many dimensions that her family appearance was approached and shared by multidisciplinary teams, gathering advanced practice nursing roles by introducing the Donabedian conceptual framework in terms of inputs, processes and outcomes. In addition, the medical team must also take care of the change of the urinary catheter, assessment of decubitus ulcers, and management of chronic disease medication to receive appropriately. For relying on the physical therapist team to help manage the right frozen shoulder, prevent attachment, to be able to manage the arms continuously. The significant approach from nurse is to help and solve problems with human kindness by nurses must use skills and expertise in health assessment, diagnosing problems, planning for nursing therapeutics and continuous evaluation. The following home visits as an independent role in providing nursing services resulting in better results, better quality of care, and reduce the problem returning to re-admission in the hospital including helping to monitor progress in solving problems, assess ability of self-care of older woman, family and community to be able to help and take care of herself more effectively.

\section{Acknowledgments}

We would like sincerely many thanks to older woman and her family, public health volunteers in Phetchaburi community Soi 7 at Bangkok, and Ramathibodi School of Nursing, Faculty of Medicine
Ramathibodi Hospital, Mahidol University that have supported and contributed this paper can be successfully accomplished.

\section{Bibliography}

1. Shaw C Wagg A. "Urinary incontinence in older adults". Medicine 45.1 (2016): 23- 27.

2. Inkoon J. "Urinary incontinence in elderly". Journal of Research in Health Sciences 22.1 (2015): 58-68.

3. Narkchuay P. "Urinary Incontinence in elderly". Royal Thai Army Medical Journal 69.1 (2016): 39-44.

4. Maipimai V, Khumtaveeporn P. "Knowledge management: caring incontinence associated dermatitis" (2014).

5. Dugger BR and Cochran A. "Urinary incontinence. In: Kristen LM editor. Gerontological nursing competencies for care. 3rd ed". Massachusetts: Jones and Bartlett Learning (2014): 544579.

6. Touhy TA and Jett KF. editors. "Ebersole and Hess' gerontological nursing and healthy aging”. St. Louis: Mosby Elsevier (2014).

7. Assantachai P. editors. "Common health problems in the elderly and prevention". 4th ed. Bangkok: Union creation Co., Ltd; (2013).

8. Larsen PD. “Lubkin's Chronic Illness: Impact and Intervention. 10th ed. Burlington". MA: Jones and Bartlett Publishers (2019).

9. Bureau of Nutrition (BoN). "The 5th Thailand nutrition and food surveillance survey 2003". Nonthaburi: Ministry of public health (2006).

10. Lawton M and Brody E. "Assessment of older people: Selfmaintaining and instrumental activities of daily living". Gerontologist 9.3 (1969): 179-186.

11. Wongpakaran N., et al. "Elderly Care Guide: formula of depression relieves". 1st ed. Nonthaburi: Openworlds publishing house Ltd (2017). 
12. Shiekh J and Yesavage J. "Geriatric Depression Scale; recent findings and development of a short version". In: Brink T, editor. Clinical gerontology: a guide to assessment and intervention. New York: Haworth Press 5.1-2 (1986): 165-173.

13. The nephrology society of Thailand. Clinical practice recommendation for the evaluation and management of chronic kidney disease in adults (2015).

14. Beeckman D., et al. "Prevention and treatment of incontinence-associated dermatitis: literature review". Journal of Advanced Nursing 65.6 (2009): 1141-1154.

15. Uppanisakorn S., et al. "Prevention and treatment of Incontinence- Associated Dermatitis (IAD) in critical patients treated in the intensive care unit of a medical ward". Thai journal of nursing council 30.2 (2015) 86-100.

16. Donabedian A. "Explorations of quality assessment and monitoring volume I: The definition of quality and approaches to its assessment". Ann Arbor, Michigan: Health administration Press. (1980).

17. Donabedian A. "The quality of care how can it be assessed?". The Journal of the American Medical Association 260.12 (1988):1743-1748.

18. Donabedian A. "An introduction to quality assurance in health care". Oxford: University Press International Journal for Quality in Health Care 15.4 (2003): 357-358.

19. Pongsiri C and Janton S. "Urinary incontinence: nurse' role". Thai Red Cross Nursing Journal 8.2 (2015): 13-25.

Volume 3 Issue 8 August 2019

(c) All rights are reserved by Supichaya Wangpitipanit., et al. 